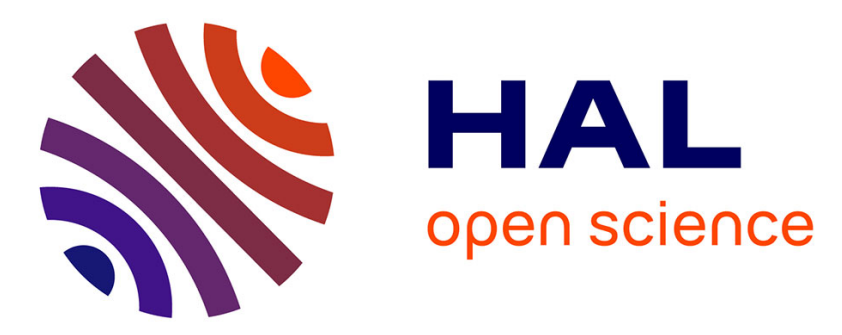

\title{
Reduced-complexity space-time block coding and decoding schemes with block linear precoding
}

Vincent Le Nir, Maryline Hélard

\section{To cite this version:}

Vincent Le Nir, Maryline Hélard. Reduced-complexity space-time block coding and decoding schemes with block linear precoding. Electronics Letters, 2003, 39, pp.1066-1068. hal-00005801

\section{HAL Id: hal-00005801 \\ https://hal.science/hal-00005801}

Submitted on 3 Aug 2005

HAL is a multi-disciplinary open access archive for the deposit and dissemination of scientific research documents, whether they are published or not. The documents may come from teaching and research institutions in France or abroad, or from public or private research centers.
L'archive ouverte pluridisciplinaire HAL, est destinée au dépôt et à la diffusion de documents scientifiques de niveau recherche, publiés ou non, émanant des établissements d'enseignement et de recherche français ou étrangers, des laboratoires publics ou privés. 


\title{
Reduced-complexity Space Time Block Coding and Decoding Schemes with Block Linear Precoding
}

\author{
V. Le Nir and M. Hélard
}

\author{
Authors' affiliation: \\ France Telecom R\&D, DMR/DDH, 4 rue du Clos Courtel, BP59, 35512 Cesson-Sevigne, France \\ Email : vincent.lenir@rd.francetelecom.fr \\ Tel.: 33299123854 (Le Nir), 33299124512 (Hélard) \\ Fax.: 33299124098
}

Key words: Space Time Block Code, Alamouti code, linear precoding, space-time diversity.

Category: Modulation \& coding 


\title{
Reduced-complexity Space Time Block Coding and Decoding Schemes with Block Linear Precoding
}

\author{
V. Le Nir and M. Hélard
}

\author{
Authors' affiliation: \\ France Telecom R\&D, DMR/DDH, 4 rue du Clos Courtel, BP59, 35512 Cesson-Sevigne, France \\ Email : \{vincent.lenir, maryline.helard\}@rd.francetelecom.com \\ Tel. : 33299123854 (Le Nir), 33299124512 (Hélard)
}

Abstract: Space-Time-Block-Coding (STBC) offers a good performance/complexity trade-off to exploit spatial diversity in multi-antenna systems. In this paper, we combine a particular linear precoder and the Alamouti STBC to improve the space-time diversity using simple linear algorithms. Our system presented with 4-transmit antennas may be applied to other STBC codes and several antenna configurations. 
Introduction: STBC was demonstrated to be a good trade-off between performance and complexity to exploit spatial diversity in multi-antenna systems. The initial 2-transmit antenna system proposed by Alamouti [1] has rate 1. Then, Tarokh [2] extended STBC to 3 or 4 transmit antennas but resulting in rate $1 / 2$ and $3 / 4$ complex orthogonal codes. Since, many studies were carried out either to find new schemes adapted to more antennas or to combine the initial Alamouti scheme to 4-transmit antennas leading to a rate 1 non orthogonal STBC $[3,4]$. In parallel, linear precoding was demonstrated to efficiently exploit time diversity in Single Input Single Output (SISO) [5] and Mutiple Input Multiple Output (MIMO) with orthogonal STBC systems [6]. In [7], the non orthogonal STBC proposed in [3] is combined with linear precoding, requiring a Maximum Likelihood (ML) decoder. New non orthogonal STBC codes are under studies as for instance in $[8,9]$. In this letter, we combine a particular linear precoding and the orthogonal Alamouti STBC to improve the space-time diversity increasing with the size of the precoding matrix. At the receiver, a simple linear decoder offers a good trade-off between performance and complexity thanks to low interference terms. We present simulation results for a 4-transmit antenna system including either a classical ML receiver or a linear one. We finally show how to extend the proposed scheme to various MIMO configurations with $N=2^{n}$ transmit antennas.

Alamouti STBC: The Alamouti STBC code for $N_{t}=2$ transmit and 1 receive antennas is represented by:

$$
\mathbf{r}=\mathbf{H} . \mathbf{s}+\mathbf{n}
$$


where $\mathbf{r}=\left[\begin{array}{ll}r_{1} & r_{2}\end{array}\right]^{T}$ is the received signal over two consecutive symbol durations, $\mathbf{s}=\left[\begin{array}{ll}s_{1} & s_{2}\end{array}\right]^{T}$ is the transmitted signal, $\mathbf{n}=\left[\begin{array}{ll}n_{1} & n_{2}\end{array}\right]^{T}$ is the additive white gaussian noise, $\mathbf{H}=\mathbf{H}_{12}=\left[\begin{array}{cc}h_{1} & h_{2} \\ -h_{2}^{*} & h_{1}^{*}\end{array}\right]$ is the equivalent channel matrix for the 2 successive symbol durations over 2 antennas, and $h_{i}$ is the channel response of transmit antenna $i$. Applying the Alamouti Maximum Ratio Combining (MRC) decoding leads to:

$$
\hat{\mathbf{s}}=\Lambda . . \mathbf{s}+\mathbf{n}^{\prime}
$$

where $\hat{\mathbf{s}}=\left[\begin{array}{ll}\hat{s}_{1} & \hat{s}_{2}\end{array}\right]^{T}$ is the estimated symbol vector after decoding, $\boldsymbol{\Lambda}=\boldsymbol{\Lambda}_{2}=\mathbf{H}_{12} \cdot \mathbf{H}_{12}^{\mathbf{H}}=\lambda_{12} \cdot \mathbf{I}_{2}$, where $(.)^{\mathrm{H}}$ stands for the transconjugate, $\mathbf{I}_{2}$ the identity $2 \times 2$ matrix, $\lambda_{12}=\left|h_{1}\right|^{2}+\left|h_{2}\right|^{2}$ and $\mathbf{n}^{\prime}=\mathbf{H}_{12}^{\mathbf{H}} \cdot \mathbf{n}$

STBC state of the art for 4-transmit antennas: Most STBC codes of rate 1 for 4-transmit antenna systems can be expressed by (1) where $\mathbf{r}=\left[\begin{array}{llll}r_{1} & r_{2} & r_{3} & r_{4}\end{array}\right]^{T}$ is the received signal and $\mathbf{s}=\left[\begin{array}{llll}s_{1} & s_{2} & s_{3} & s_{4}\end{array}\right]^{T}$ the transmitted one. For the STBC proposed in $[3,4], \mathbf{H}$ is a $4 \times 4$ matrix equals to either $\mathbf{H}=\left[\begin{array}{cc}\mathbf{H}_{12} & \mathbf{H}_{34} \\ -\mathbf{H}_{34}^{*} & \mathbf{H}_{12}^{*}\end{array}\right]$ or $\mathbf{H}=\left[\begin{array}{ll}\mathbf{H}_{12} & \mathbf{H}_{34} \\ \mathbf{H}_{34} & \mathbf{H}_{12}\end{array}\right] . \quad \mathbf{H}_{i j}=\left[\begin{array}{cc}h_{i} & h_{j} \\ -h_{j}^{*} & h_{i}^{*}\end{array}\right]$ is the equivalent channel matrix for 2 successive symbol durations over 2 antennas $\mathrm{i}$ and $\mathrm{j}, h_{i}$ and $h_{j}$ are the channel responses of transmit antenna $i$ and $j$ respectively. In [7], the linear precoding is applied to the first previous matrix. In fact, all these STBC require ML decoders due to high interference terms. 
The proposed scheme for 4-transmit antennas: In our 4-transmit antenna system, we first apply Alamouti STBC alternatively to antennas 1 and 2 and then to antennas 3 and 4 . Thus, we obtain the following equivalent matrix:

$$
\mathbf{H}=\left[\begin{array}{cc}
\mathbf{H}_{12} & \mathbf{0} \\
\mathbf{0} & \mathbf{H}_{34}
\end{array}\right]
$$

of size $4 x 4$. At the reception, we get (2) with $\boldsymbol{\Lambda}=\boldsymbol{\Lambda}_{4}=\mathbf{H} \cdot \mathbf{H}^{\mathbf{H}}=\operatorname{diag}\left(\lambda_{12}, \lambda_{12}, \lambda_{34}, \lambda_{34}\right)$ and $\lambda_{i j}=\left|h_{i}\right|^{2}+\left|h_{j}\right|^{2}$ leading to a 2 channel diversity order. Before this space-time code, we apply a linear precoding represented by a $L x L$ unitary matrix $\boldsymbol{\Theta}_{\mathbf{L}}=\sqrt{\frac{2}{L}} \cdot\left[\begin{array}{cc}\Theta_{L / 2} & \Theta_{L / 2} \\ \Theta_{L / 2} & -\Theta_{L / 2}\end{array}\right]$ based on Hadamard construction with $\boldsymbol{\Theta}_{2}=\left[\begin{array}{cc}e^{i \theta_{1}} \cos \eta & e^{i \theta_{2}} \sin \eta \\ -e^{-i \theta_{2}} \sin \eta & e^{-i \theta_{1}} \cos \eta\end{array}\right]$ the general matrix of the $S U(2)$ group $\left(\boldsymbol{\Theta}_{2}^{-1}=\boldsymbol{\Theta}_{2}^{H}\right.$ and $\left.\operatorname{det} \boldsymbol{\Theta}_{2}=1\right)$ where $\eta, \theta_{1}$ and $\theta_{2}$ are parameters to be further optimized.

For instance, for $L=4$ and $\boldsymbol{\Theta}_{2}=\frac{1}{\sqrt{2}}\left[\begin{array}{cc}1 & 1 \\ -1 & 1\end{array}\right]$, we obtain the global transmission and reception system described by following matrix: $\mathbf{A}_{4}=\boldsymbol{\Theta}_{4} \cdot \boldsymbol{\Lambda}_{4} \cdot \boldsymbol{\Theta}_{4}^{H}=\frac{1}{2}\left[\begin{array}{cccc}\lambda_{12}+\lambda_{34} & 0 & \lambda_{12}-\lambda_{34} & 0 \\ 0 & \lambda_{12}+\lambda_{34} & 0 & \lambda_{12}-\lambda_{34} \\ \lambda_{12}-\lambda_{34} & 0 & \lambda_{12}+\lambda_{34} & 0 \\ 0 & \lambda_{12}-\lambda_{34} & 0 & \lambda_{12}+\lambda_{34}\end{array}\right]$ where $\boldsymbol{\Theta}_{4}^{H} \quad$ is the linear decoding. Diagonal elements of $\mathbf{A}_{4}$ are all equal to $\frac{1}{2} \sum_{l=1}^{L=4}\left|h_{l}\right|^{2}$. Therefore, thanks to linear precoding, the exploited channel diversity order increases from 2 to 4 . Moreover, interference terms are either null or proportional to $\frac{1}{2}\left(\sum_{l=1}^{2}\left|h_{l}\right|^{2}-\sum_{l=3}^{4}\left|h_{l}\right|^{2}\right)$ and smaller than those of systems described in the bibliography. 
If we apply a $L x L$ precoding matrix to our 4 transmit antenna system, at the reception, we get (2) with $\boldsymbol{\Lambda}=\boldsymbol{\Lambda}_{L}=\mathbf{H} . \mathbf{H}^{\mathbf{H}}=\operatorname{diag}\left(\lambda_{12}, \lambda_{12}, \ldots, \lambda_{(L-1) L}, \lambda_{(L-1) L}\right)$ where $\mathbf{H}$ is a $L x L$ matrix expressed by: $\mathbf{H}=\left[\begin{array}{ccc}\mathbf{H}_{12} & 0 & 0 \\ 0 & \ddots & 0 \\ 0 & 0 & \mathbf{H}_{(L-1) L}\end{array}\right]$

Thus, the global transmission/reception scheme is given by:

$$
\mathbf{A}_{\mathbf{L}}=\boldsymbol{\Theta}_{\mathbf{L}} \cdot \boldsymbol{\Lambda}_{L} \cdot \boldsymbol{\Theta}_{\mathbf{L}}^{H}
$$

All the diagonal elements of $\mathbf{A}_{\mathbf{L}}$ are equal to $\frac{2}{L} \sum_{l=1}^{L}\left|h_{l}\right|^{2}$ while interference terms are equivalent to $\frac{2}{L}\left(\sum_{l=1}^{L / 2}\left|h_{l}\right|^{2}-\sum_{l=L / 2+1}^{L}\left|h_{l}\right|^{2}\right)$. Moreover, for flat independent Rayleigh channels, the diagonal terms tend to a non-centered gaussian law while the interference terms tend to a centered gaussian law when $\mathrm{L}$ increases.

We can note that $\mathbf{H}$ is the same for all $N_{t}=2^{n}$ antenna systems, with $n \geq 1$, when $L=2^{m}$ with $m \geq n$. Since the global formulation (4) does not depend on $N_{t}$, our linear precoding can be applied to several multiple antenna systems and various transmit antennas $N_{t}=2^{n}$ with $1 \leq n \leq m$. Thus, the presented STBC for four transmit antennas with linear precoding of size $L x L$ is similar to the STBC for two transmit antennas with linear precoding of size $\frac{L}{2} \times \frac{L}{2}$ with uncorrelated channels between transmit and receive antennas.

Simulations results: We carried out simulations over flat independent Rayleigh channels. Depending on the choice of $\boldsymbol{\Theta}_{2}$ based matrix, we have demonstrated by simulation that the best performance leads to interferer terms either pure real or pure imaginary which is obtained for 
instance with $\eta=\frac{\pi}{4}, \theta_{1}=\frac{5 \pi}{4}$ and $\theta_{2}=\frac{3 \pi}{4}$ and a Minimum Mean Square Error (MMSE) equalizer for the STBC decoding.

In Figure 1, we present BER performance obtained for systems with $N_{t}=4$ transmit and $N_{r}=1$ receive antennas, with either a linear $L I N$ or a $M L$ decoder, and different $L x L$ size of precoding matrix as presented in (4). We observe that the specified system with $L=4$ leads to better performance than the sole Alamouti scheme without precoding as described in (3). In fact, all diagonal terms $\frac{1}{2} \sum_{l=1}^{L=4}\left|h_{l}\right|^{2}$ follow a $\chi_{8}^{2}$ chi-square law while the interference terms in $\frac{1}{2}\left(\sum_{l=1}^{2}\left|h_{l}\right|^{2}-\sum_{l=3}^{4}\left|h_{l}\right|^{2}\right)$ follow a law of $\chi_{4}^{2}$ difference. We can notice that for $L=4$, the penalty when using linear decoder denoted $L I N$ instead of the $M L$ decoder is very small. We observed that this penalty diminishes when $\mathrm{L}$ increases thanks to the form of interference terms null or of the form $\frac{2}{L}\left(\sum_{l=1}^{L / 2}\left|h_{i}\right|^{2}-\sum_{l=L / 2+1}^{L}\left|h_{i}\right|^{2}\right)$ and the curves match at $L=64$. We also see that the diversity increases with L following a $\chi_{2 L}^{2}$ law for diagonal terms. In fact, the slope of the curve corresponding to $L=64$ is almost parallel to the gaussian curve. The curve $L=16$ could have been obtained with $2,4,8$ or 16 antennas under the assumption of independent Rayleigh channels every 2 symbol durations. The performance of the system with $L=64$ is very close to the asymptotic performance.

Conclusion: The proposed combination of linear precoding and Alamouti STBC applied by block of 2 antennas leads to an efficient exploitation of space-time diversity that increases with the length of the precoding matrix at a linear cost of complexity. In fact, our system described with 4transmit antennas may be applied to other STBC codes and several antenna configurations. 
Moreover, the greater L, the smaller the interference terms. Thus, a simple linear decoder is sufficient when using a STBC combined with linear precoding.

\section{References:}

1. ALAMOUTI, S. 'A Simple Transmit Diversity Technique for Wireless Communications', IEEE J. Sel. Areas Comm., 1998, 16, (8), pp. 1451-1458.

2. TAROKH, V., JAFARKHANI, H. and CALDERBANK, A. R, 'Space-time block codes from orthogonal designs', IEEE Trans. on Information Theory, 1999, 45, (5), pp. 1456-1467.

3. JAFARKHANI, H.'A quasi-Orthogonal Space-Time Block Code', IEEE Trans. Comm., 2001, 49, (1), pp1-4.

4. TIRKKONEN, O., BOARIU, A. and HOTTINEN, A., 'Minimal non-orthogonality rate one spacetime block code for 3+ Tx antennas', ISSSTA, 2000, pp 429-432.

5. BOUTROS, J., 'Signal Space Diversity: A Power and Bandwidth Efficient Diversity Technique for the Rayleigh Fading Channel', IEEE Trans. On Information Theory, 1998, 44, (4), pp.1453-1467.

6. STAMOULIS, A., LIU, Z. and GIANNAKIS, G. B., 'Space-Time Block-Coded OFDMA With Linear Precoding for Multirate Services', IEEE Trans. on Signal Process., 2002, 50, (1).

7. DA SILVA and CORREIA, A., 'Space Time Block Coding for 4 Antennas with Coding Rate 1', IEEE Int. Symp. on Spread-Spectrum Tech. and Appl., Prague, Czech Republic, 2002.

8. DAMEN, M. O., ABED-MERAIM, K. and BELFIORE, J.-C., 'Diagonal Algebraic Space-Time Block Codes', IEEE Trans. Inf. Theory, 2002, 48, (3), pp 628-626.

9. XIN, Y., WANG and GIANNAKIS, Z.G., 'Space-Time Constellation-Rotating Codes Maximizing Diversity and Coding Gains', GLOBECOM, San Antonio, 2001, pp 455-459. 


\section{Figure captions :}

Fig. 1: Bit Error Performance of different 4-transmit antenna systems including STBC and linear precoding. 
Figure 1

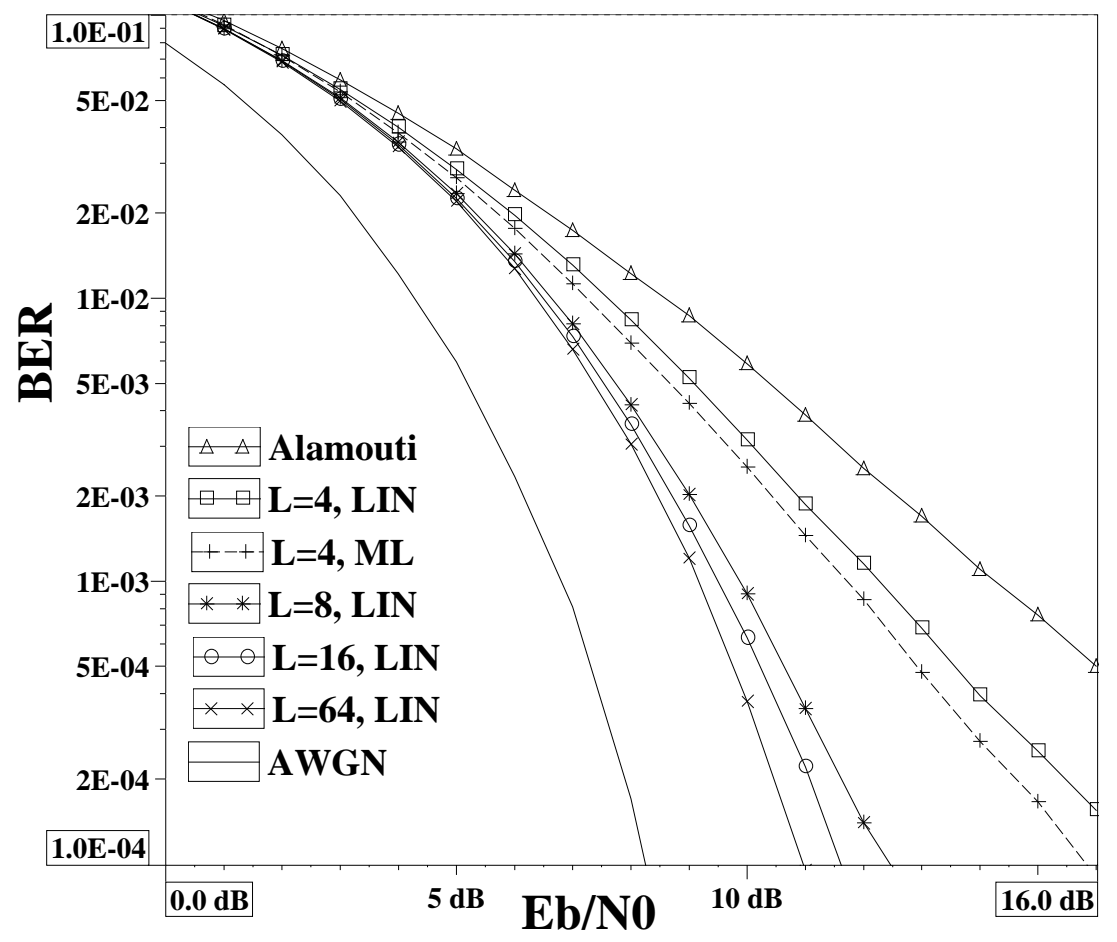

